\title{
An intraoperative test device for aortic valve repair
}

Ignacio G. Berra, MD, ${ }^{a}$ Peter E. Hammer, PhD, ${ }^{\mathrm{b}}$ Sebastian Berra, ${ }^{\mathrm{c}}$ Alfredo Oscar Irusta, ${ }^{\mathrm{d}}$ Seok Chang Ryu, Douglas P. Perrin, PhD, ${ }^{b}$ Nikolay V. Vasilyev, MD, ${ }^{b}$ Carlos Javier Cornelis, MD, Pablo Garcia Delucis, MD, ${ }^{\mathrm{a}}$ and Pedro J. del Nido, $\mathrm{MD}^{\mathrm{b}}$

\section{ABSTRACT}

Objective: Aortic valve repair is currently in transition from surgical improvisation to a reproducible operation and an option for many patients with aortic regurgitation. Our research efforts at improving reproducibility include development of methods for intraoperatively testing and visualizing the valve in its diastolic state.

Methods: We developed a device that can be intraoperatively secured in the transected aorta allowing the aortic root to be pressurized and the closed valve to be inspected endoscopically. Our device includes a chamber that can be pressurized with crystalloid solution and ports for introduction of an endoscope and measuring gauges. We show use of the device in explanted porcine hearts to visualize the aortic valve and to measure leaflet coaptation height in normal valves and in valves that have undergone valve repair procedures.

Results: The procedure of introducing and securing the device in the aorta, pressurizing the valve, and endoscopically visualizing the closed valve is done in less than 1 minute. The device easily and reversibly attaches to the aortic root and allows direct inspection of the aortic valve under conditions that mimic diastole. It enables the surgeon to intraoperatively study the valve immediately before repair to determine mechanisms of incompetence and immediately after the repair to assess competence. We also show its use in measuring valve leaflet coaptation height in the diastolic state.

Conclusions: This device enables more relevant prerepair valve assessment and also enables a test of postrepair valve competence under physiological pressures. (J Thorac Cardiovasc Surg 2019;157:126-32)

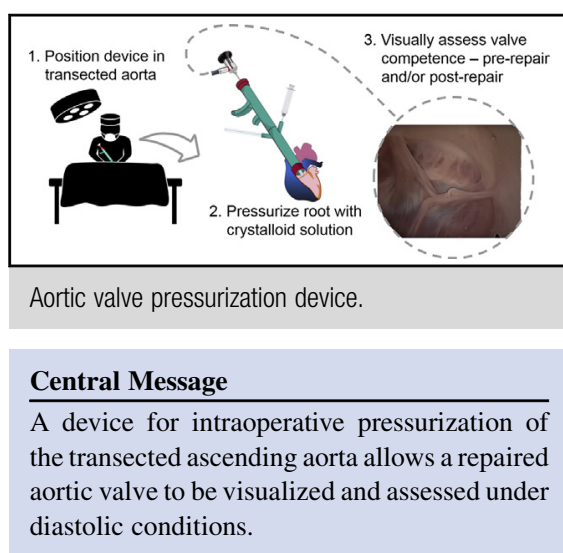

\section{Perspective}

Our device allows intraoperative visualization of the pressurized aortic valve-prerepair, to see the mechanism of valve dysfunction, and postrepair, to test competence. Repairs can be tested quickly and without coming off bypass. This capability could raise the success rate of aortic valve repair, leading to higher repair rates and encouraging less experienced surgeons to attempt repair.

See Editorial Commentary page 133.

See Editorial page 124.
In moderate to severe aortic valve disease, surgery is required to prevent adverse cardiac remodeling that can ultimately progress to heart failure and death. In children,

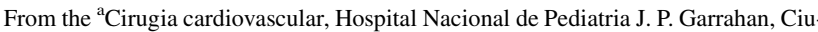
dad Autonoma de Buenos Aires, Argentina; ${ }^{b}$ Department of Cardiac Surgery, Boston Children's Hospital, Boston, Mass; ${ }^{\mathrm{c} C}$ atedra de control, Universidad de Buenos Aires, Ciudad Autonoma de Buenos Aires, Argentina; ' ${ }^{\mathrm{L}}$ ew insumos e Innovaciones S.A., Moron, Buenos Aires, Argentina; and ${ }^{\mathrm{e}}$ Mechanical Engineering, Texas A\&M University, College Station, Tex.

Read at The American Association for Thoracic Surgery Aortic Symposium 2018, New York, New York, April 26-27, 2018.

Drs Berra and Hammer contributed equally to this work

Received for publication April 24, 2018; revisions received June 22, 2018; accepted for publication July 6, 2018.

Address for reprints: Ignacio G. Berra, MD, Cirugia cardiovascular, Hospital Nacional de Pediatria J. P. Garrahan, Ciudad Autonoma de Buenos Aires, Argentina (E-mail: nachoberra@yahoo.com).

0022-5223/\$36.00

Copyright (c) 2018 by The American Association for Thoracic Surgery

https://doi.org/10.1016/j.jtcvs.2018.07.113
}

surgical repair of the valve is usually preferred over valve replacement. ${ }^{1,2}$ However, aortic valve repair is technically challenging and is currently in transition from surgical improvisation to a reproducible operation and an option for many patients with aortic valve disease. A major challenge to surgeons during aortic valve repair is that the repair is typically performed in the transected, unpressurized aortic root. However, this state is nonphysiological, because the in vivo pressure in the

Scanning this QR code will take you to the article title page to access supplementary information. To view the AATS Webcast, see the URL next to the webcast thumbnail. 


\section{Abbreviations and Acronyms \\ $\mathrm{AR}=$ aortic regurgitation \\ $\mathrm{STJ}=$ sinotubular junction}

aortic root continuously oscillates between systolic and diastolic pressures. This makes it difficult for the surgeon to intraoperatively assess valve dysfunction before repair and to predict valve competence after repair solely on the basis of intraoperative, unpressurized configurations. In mitral valve repair, it is common to inject saline into the left ventricle with a bulb syringe to test valve competence. ${ }^{3}$ There is no similar test in use for the aortic valve, although the concept has been proposed. ${ }^{4}$

Our research efforts at improving the reproducibility of aortic valve repair techniques have included development of a device and an experimental method to pressurize and endoscopically visualize the closed aortic valve during the intraoperative period while the aorta is transected. The purpose of the device is twofold: first, it allows a regurgitant aortic valve to be inspected under typical diastolic conditions at the start of the repair to better understand mechanisms of aortic regurgitation (AR). Second, it enables the surgeon to postoperatively assess the efficacy of repair and the robustness of valve closure. For a measure of the robustness of valve closure, we use the amount of overlap of the leaflets in the valve center under peak load. Surgeons have noted that in the center of the valve, the amount of overlap, referred as central coaptation length, is an important measure of valve function and a strong predictor of durability of aortic valve repair. ${ }^{5,6}$

In this report, we describe the design of the device and its use to visualize the aortic valve under typical diastolic pressures (Video 1). We then show how the device can be used to measure valve coaptation length, and describe 2 methods for doing so. Finally, we describe use of the device in 3 different studies on explanted porcine hearts that assess: (1) normal aortic valves $(\mathrm{n}=5)$; (2) repaired aortic valves in a model of AR ( $\mathrm{n}=3)$; and (3) aortic valves that have been reconstructed using the method of Ozaki and colleagues $(n=3){ }^{7}$

\section{METHODS}

\section{Device Description and Use}

We have developed a device that can be used intraoperatively to subject the aortic root to typical diastolic pressures, allowing the valve to be visualized in its physiological closed state. This device is a 2-piece cylindrical tube with length of $26 \mathrm{~cm}$, outside diameter of $2 \mathrm{~cm}$, and inside diameter of $1.5 \mathrm{~cm}$ (Figure 1). The proximal section is a working channel with inlet and outlet valves, and the distal section is a pressurization chamber. The pressurization chamber has 2 side ports. One side port is connected via a quarter-inch tubing with a $60-\mathrm{mL}$ syringe and a pressure sensor to enable pressure to be monitored and regulated. The other side port can be used to introduce measurement tools. This device was printed on a Stratasys Object 30 Prime 3D printer (Eden Prairie, Minn) with a super clear resin.

To use the device in the explanted porcine hearts, the aorta was transected $5 \mathrm{~cm}$ above the sinotubular junction (STJ) to ensure that the

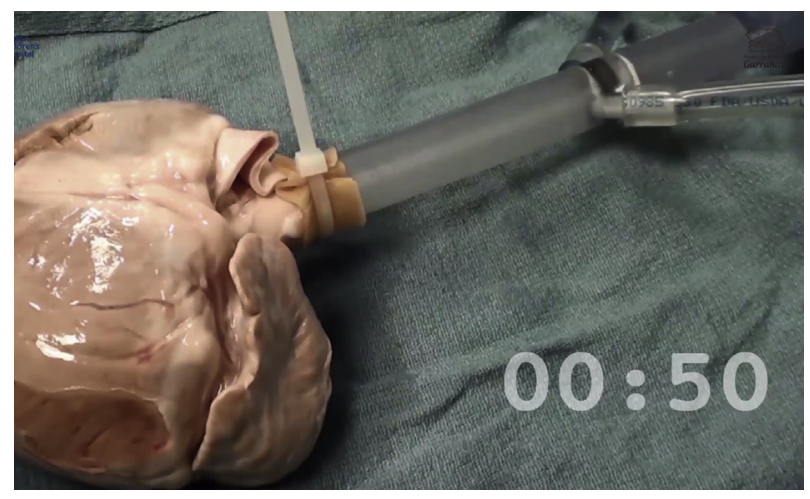

VIDEO 1. This animation shows positioning of the device in the transected aorta, insertion of endoscope through the device, pressurization of the device chamber and valve root with crystalloid solution, and introduction of a gauge for measuring valve coaptation height. Video available at: https://www.jtcvs.org/article/S0022-5223(18)32820-4/fulltext.

approximately $30-\mathrm{mm}$ diameter valve root is not distorted when cinching it around the 20-mm diameter device. (Note that for hearts well matched in size to the device, the aortotomy can be within $1 \mathrm{~cm}$ of the STJ.) The pressurization device is inserted into the lumen of the transected aorta, and a size 0 silk suture or a plastic cable tie is used to cinch the aorta around the device. To occlude the coronary ostia, tourniquets were applied around the coronary arteries. A $60-\mathrm{mL}$ syringe was used to inject crystalloid solution into the aortic root via the tubing and device side port. Air bubbles must be bled from the aortic root and pressurized chamber so as not to interfere with observation of the valve structures. A rigid 7-mm diameter endoscope was then introduced through the upper working channel and advanced into the pressurization chamber to allow visualization of the valve and aortic root.

\section{Methods for Measuring Valve Coaptation Length}

We have developed and used 2 methods to perform the measurement of valve coaptation length while the valve is being pressurized and visualized. The first method uses a flexible plastic scale, 60-mm long, 2-mm wide, and marked in millimeters. While the valve is pressurized and endoscope is in

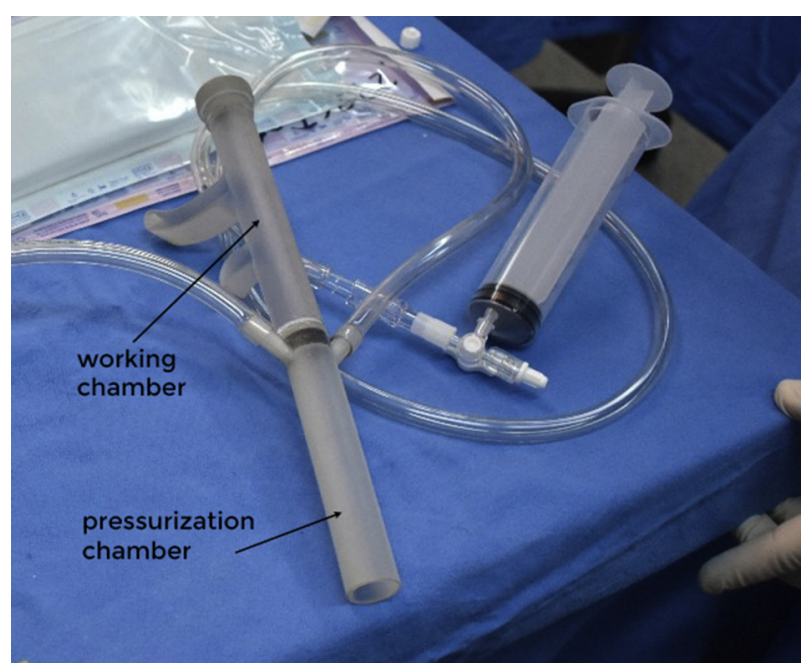

FIGURE 1. The aortic valve pressurization device consists of a pressurization chamber that is secured inside the transected aorta, a working chamber through which an endoscope is introduced, and side ports for delivering pressurized perfusate and introduction of measurement gauges. 
place, the scale is advanced through the device side port into the pressurization chamber, into the valve root, and then between the leaflets of the closed valve. The endoscope is positioned at the top of the coapted leaflets and advanced between the leaflets, counting the marks along the scale until the leaflets are pushed open. This allows quantification of the distance over which the leaflets are in contact. The scale also makes it possible to measure any discrepancy that exists between 2 coapted leaflets (ie, the distance by which the free edge of one leaflet extends past the free edge of an adjacent leaflet when closed). These metrics of the conformation of the closed valve provide important information for the surgeon in determining the best surgical strategy to achieve a normal aortic valve configuration and durable repair.

The second method for measuring coaptation length uses a metal rod with a $1-\mathrm{cm}$ diameter disk at its distal end. The rod is covered by a transparent plastic tube of the same length that is free to slide along it. To measure valve coaptation length, the plastic tube is first slid until the tube completely covers the rod, then the rod and tube are advanced until the disk is on the ventricular side of the closed valve. The rod and tube are then retracted until the disk is in contact with the ventricular surface of the leaflets of the closed valve. Then, under endoscopic vision, the transparent tube is slid upward (away from the disk) until its end just becomes visible at the top of the leaflet coaptation zone. The length of the coaptation zone can now be quantified by measuring the distance beyond which the tube extends past the proximal end of the rod (Figure 2).

\section{Study 1: Coaptation in the Normal (Porcine) Aortic Valve}

In explanted hearts from 5 Landrace pigs weighing between 70 and $80 \mathrm{~kg}$, we first measured anatomical features of the valves in the flaccid state, including the STJ diameter, the width of all leaflets, and the height of all leaflets. We then introduced the aortic pressurization device as described previously and assessed the conformation of the aortic valve under pressure of $80 \mathrm{~mm} \mathrm{Hg}$ using a 7-mm diameter rigid endoscope. We measured the valve coaptation length using the rod and disk method described previously.
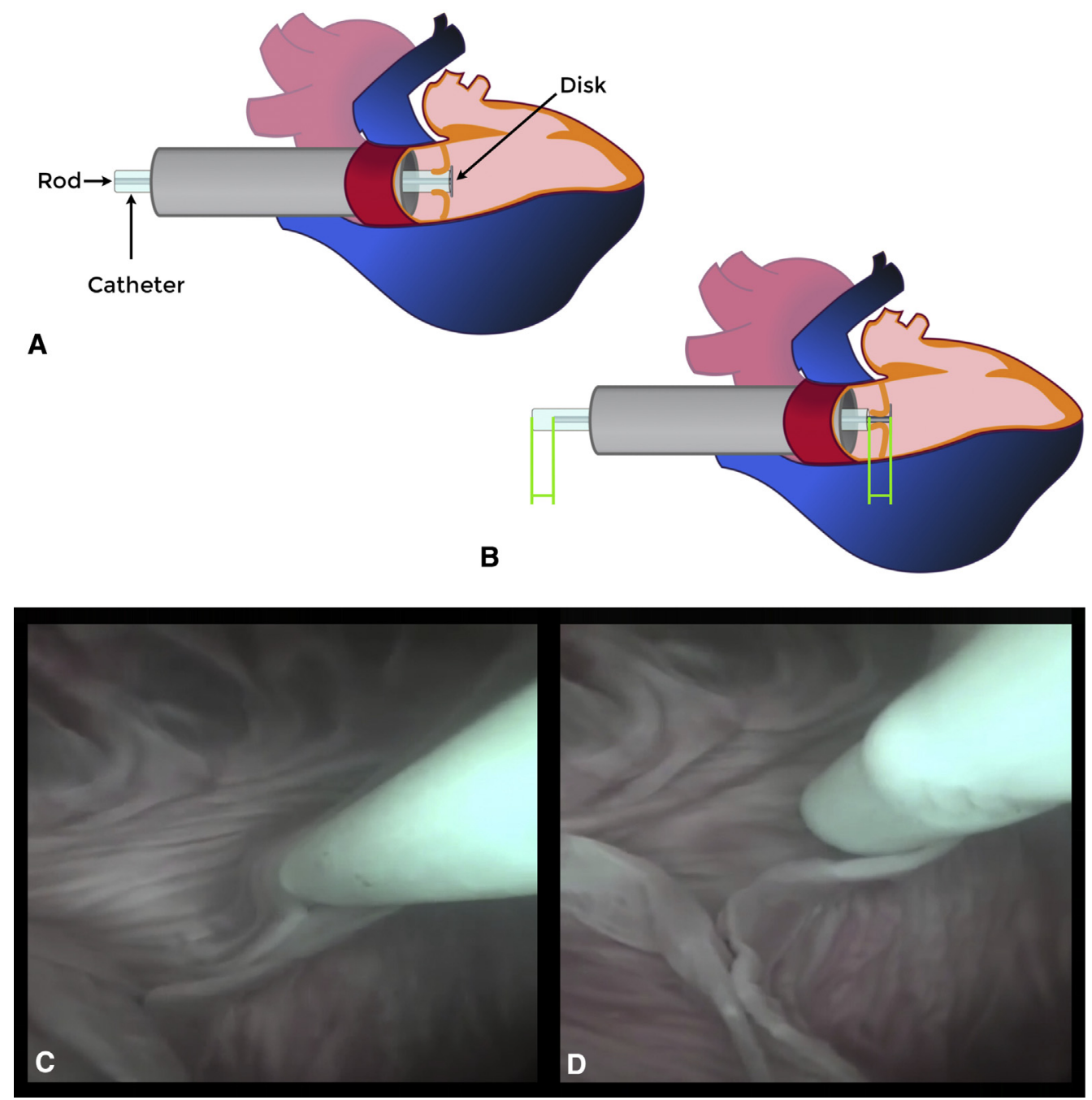

FIGURE 2. Leaflet coaptation can be measured using a gauge consisting of a thin metal rod with a 1-cm diameter disk at one end. A plastic tube covers the rod and can be slid along it. Starting with the tube completely covering the rod and the disk pulled flush with the ventricular surface of the closed valve (A), the tube is pulled back until its distal end is just visible above the free edges of the coapted leaflets (B). The coaptation height can be measured at the proximal end of the gauge as the distance from the end of the rod to the end of the tube (dimension shown in green). Starting with the tube completely covering the rod and the disk pulled flush with the ventricular surface of the closed valve $(C)$, the tube is pulled back until its distal end is just visible above the free edges of the coapted leaflets (D). 
TABLE 1. Valve dimensions in normal porcine hearts

\begin{tabular}{|c|c|c|c|c|c|c|c|c|c|c|c|c|c|c|c|}
\hline- & \multicolumn{3}{|c|}{ Pig 1} & \multicolumn{3}{|c|}{ Pig 2} & \multicolumn{3}{|c|}{ Pig 3} & \multicolumn{3}{|c|}{ Pig 4} & \multicolumn{3}{|c|}{ Pig 5} \\
\hline Sinotubular junction, $\mathrm{mm}$ & \multicolumn{3}{|c|}{30} & \multicolumn{3}{|c|}{31} & \multicolumn{3}{|c|}{33} & \multicolumn{3}{|c|}{30} & \multicolumn{3}{|c|}{33} \\
\hline Coaptation length, mm & \multicolumn{3}{|c|}{5} & \multicolumn{3}{|c|}{6} & \multicolumn{3}{|c|}{7} & \multicolumn{3}{|c|}{5} & \multicolumn{3}{|c|}{5} \\
\hline \multirow[t]{2}{*}{ Leaflet width, mm } & $\mathrm{NC}$ & $\mathrm{RC}$ & $\mathrm{LC}$ & $\mathrm{NC}$ & $\mathrm{RC}$ & LC & $\mathrm{NC}$ & $\mathrm{RC}$ & LC & $\mathrm{NC}$ & $\mathrm{RC}$ & $\mathrm{LC}$ & $\mathrm{NC}$ & $\mathrm{RC}$ & $\mathrm{LC}$ \\
\hline & 22 & 25 & 24 & 23 & 25 & 23 & 21 & 26 & 24 & 22 & 25 & 25 & 21 & 25 & 24 \\
\hline \multirow[t]{2}{*}{ Leaflet length, mm } & $\mathrm{NC}$ & $\mathrm{RC}$ & $\mathrm{LC}$ & $\mathrm{NC}$ & $\mathrm{RC}$ & LC & $\mathrm{NC}$ & $\mathrm{RC}$ & $\mathrm{LC}$ & $\mathrm{NC}$ & $\mathrm{RC}$ & $\mathrm{LC}$ & $\mathrm{NC}$ & $\mathrm{RC}$ & LC \\
\hline & 16 & 16 & 16 & 17 & 17 & 17 & 17 & 17 & 17 & 17 & 17 & 17 & 17 & 17 & 17 \\
\hline
\end{tabular}

$N C$, Noncoronary; $R C$, right coronary; $L C$, left coronary.

\section{Study 2: Repaired Aortic Valves in a Model of AR}

In a different group of 3 explanted porcine hearts, we created an AR model by surgically reducing the right coronary leaflet and a portion of the right coronary root. ${ }^{8}$ The height and width of all leaflets was measured after creation of the regurgitation model, and the valves were tested with the pressurization device to confirm the presence of regurgitation. The regurgitant valves were then repaired by resection or plicating a 10-mm wide section from the left coronary sinus, extending from the STJ to the valve annulus. ${ }^{8}$ The pressurization device was then introduced, and the conformation of the aortic valve was evaluated by applying a pressure of $80 \mathrm{~mm} \mathrm{Hg}$ and visualizing the valve endoscopically. Coaptation length was measured using the method involving the flexible plastic scale.

\section{Study 3: Aortic Valves Reconstructed Using Pericardium}

In a third group of explanted hearts, we performed aortic valve reconstruction in 3 hearts using the sizers and templates developed by Ozaki and colleagues, ${ }^{7}$ constructing leaflets from patches of glutaraldehyde fixed bovine pericardium measuring between 27 and $29 \mathrm{~mm}$ of width and length for all leaflets. The pressurization device was introduced, and the

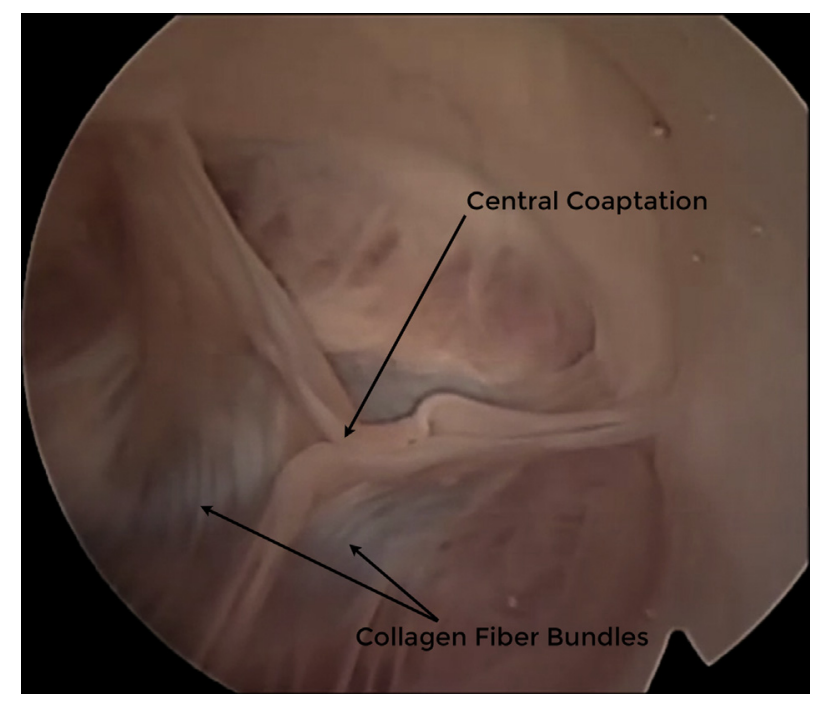

FIGURE 3. Endoscopic view of normal aortic valve under diastolic conditions showing leaflet coaptation near the valve center and a normal pattern of collagen fiber bundles. conformation of the aortic valve at a pressure of $80 \mathrm{~mm} \mathrm{Hg}$ was endoscopically visualized. Coaptation length was measured using the method involving the flexible plastic scale.

\section{RESULTS}

The procedure of performing the pressurization and endoscopic visualization of the closed valve is done in less than 1 minute. The device easily and reversibly attaches to the aortic root and allows direct inspection of the aortic valve under diastolic pressures immediately before and after the repair. It enables qualitative assessment of valve competence, symmetry of the closed valve, and leaflet pliability, and it also enables quantitative determination of valve leaflet coaptation length in the diastolic state. In the healthy porcine valves that we observed, we were also able to discern the patterns of the collagen fiber bundles within the leaflets.

In study 1, we observed normal, competent valves in the diastolic state and noted the slight skew of the central point of coaptation toward the noncoronary leaflet. Leaflet measurements also indicated that the noncoronary leaflet tends to be the smallest of the 3 leaflets in a given valve (Table 1). Measurement of coaptation length yielded a mean value of approximately $6 \mathrm{~mm}$ in valves with STJ diameter of approximately $30 \mathrm{~mm}$. All valves exhibited normal coaptation near the valve center and a normal pattern of collagen fiber bundles under diastolic pressure conditions (Figure 3). Prolapse of the leaflets was not observed and the metal disk of the coaptation height measuring gauge could be seen.

In study 2, our models of AR exhibited considerable asymmetry, in the leaflet dimensions (Table 2) and in the skew of the central coaptation point toward the right coronary leaflet. After surgical repair of the valves by resecting or plicating a portion of the left coronary sinus, we endoscopically inspected the valves and determined that all were competent. We measured the central coaptation length using the method with the flexible plastic scale (Figure 4) 
TABLE 2. Valve dimensions in porcine model of aortic root resection

\begin{tabular}{|c|c|c|c|c|c|c|c|c|c|}
\hline 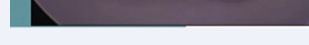 & & Pig 1 & & \multicolumn{3}{|c|}{ Pig 2} & \multicolumn{3}{|c|}{ Pig 3} \\
\hline Sinotubular junction, mm & \multicolumn{3}{|c|}{30} & \multicolumn{3}{|c|}{27} & \multicolumn{3}{|c|}{29} \\
\hline Coaptation length, mm & \multicolumn{3}{|c|}{1} & \multicolumn{3}{|c|}{4} & \multicolumn{3}{|c|}{2} \\
\hline \multirow[t]{2}{*}{ Leaflet width, mm } & $\mathrm{NC}$ & $\mathrm{RC}$ & $\mathrm{LC}$ & $\mathrm{NC}$ & $\mathrm{RC}$ & $\mathrm{LC}$ & $\mathrm{NC}$ & $\mathrm{RC}$ & LC \\
\hline & 22 & 16 & 24 & 22 & 16 & 26 & 23 & 17 & 25 \\
\hline \multirow[t]{2}{*}{ Leaflet length, mm } & $\mathrm{NC}$ & $\mathrm{RC}$ & $\mathrm{LC}$ & $\mathrm{NC}$ & $\mathrm{RC}$ & $\mathrm{LC}$ & $\mathrm{NC}$ & $\mathrm{RC}$ & LC \\
\hline & 16 & 16 & 16 & 16 & 16 & 16 & 16 & 16 & 16 \\
\hline
\end{tabular}

$N C$, Noncoronary; $R C$, right coronary; $L C$, left coronary.

and observed a mean central coaptation length of $2 \mathrm{~mm}$ across the 3 specimens (Table 2).

In study 3, all 3 valves exhibited coaptation of the 3 leaflets that was central, and the mean value of coaptation height was $19 \mathrm{~mm}$ (Table 3). Endoscopic visualization revealed considerable redundancy of the leaflet free edges under pressurization of $80 \mathrm{~mm} \mathrm{Hg}$ (Figure 5).

\section{DISCUSSION}

Aortic valve repair is hampered by the nonphysiological state of the valve during surgery and could be greatly facilitated by a means of intraoperatively inspecting the valve under pressure. This capability would allow the surgeon to directly see the location and mechanism of regurgitation prerepair and to choose an appropriate repair strategy. Perhaps more importantly, it could also allow a repair to be immediately tested and rerepaired in an iterative process

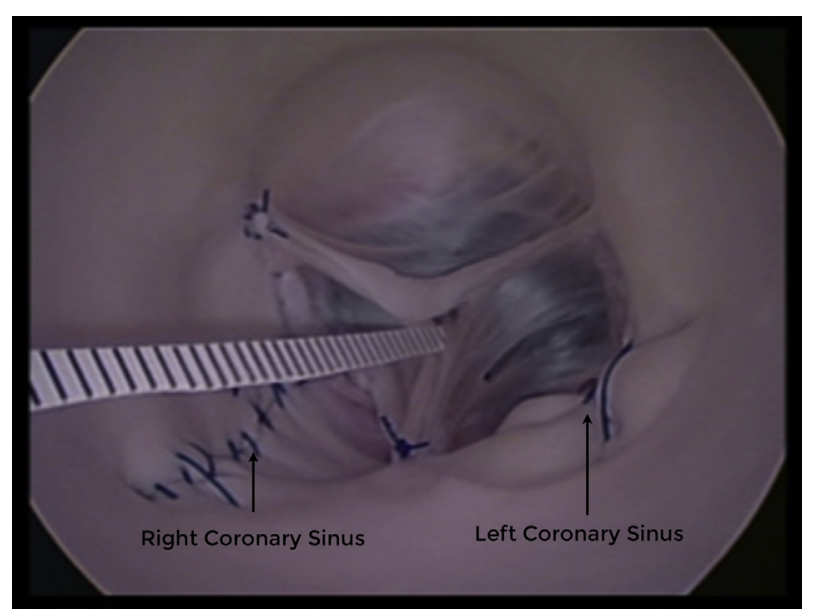

FIGURE 4. Endoscopic view showing porcine model of aortic regurgitation in which the right coronary sinus and leaflet have been surgically reduced. We then repaired the valve by plicating a portion of the left coronary sinus, and we measured central coaptation length in the repaired valve using the flexible plastic scale. without coming off bypass. Such a pressure test is widely used in mitral valve repair and likely plays a role in the high rates of repair of these valves. It is worth noting that an intraoperative pressurization test is perhaps more appropriate for the aortic valve because the in vivo valve is passively loaded during diastole. This is not the case for mitral valve which, in vivo, is closed and loaded when the ventricle and papillary muscles contract, producing different chamber and valve geometry than during passive pressure testing.

Our experimental results show the use of our pressurization device to inspect several important features of the loaded valve. The most important feature is the absence of leak at physiological diastolic pressures, which is the ultimate goal of valve repair for AR. Our device lets the surgeon detect AR by directly viewing any regurgitant orifice and by noting the need for continuous infusion from the syringe to maintain constant pressure across the valve. Conversely, absence of AR can be inferred by the absence of any gap centrally and along the margins where the leaflets meet, as we have shown in the endoscopic images (Figures 3-5). Absence of AR can also be inferred from the ability of the valve root to maintain constant pressure without continuous infusion. A second important feature of aortic valve repair is central coaptation length, and we showed measurement of this dimension in a repair that preserves the native leaflets as well as in a repair that involves complete replacement of all 3 leaflets with graft material. The method used in the latter study uses very tall leaflet grafts intended to result in coaptation heights that are considerably greater than in the normal valve. ${ }^{9}$ Use of our device and method allowed quantification of this feature under typical diastolic pressure, confirming the very large coaptation height. Not only is the pressurization device helpful for assessing closure of reconstructed leaflets in a native root, but it could also be used in the opposite case: native leaflets in a reconstructed root, as in a valve-sparing root replacement. Although we have not 
TABLE 3. Valve dimensions in porcine model of Ozaki repair

\begin{tabular}{|c|c|c|c|c|c|c|c|c|c|}
\hline & & Pig 1 & & \multicolumn{3}{|c|}{ Pig 2} & \multicolumn{3}{|c|}{ Pig 3} \\
\hline Sinotubular junction, $\mathrm{mm}$ & \multicolumn{3}{|c|}{29} & \multicolumn{3}{|c|}{28} & \multicolumn{3}{|c|}{27} \\
\hline Coaptation length, mm & \multicolumn{3}{|c|}{18} & \multicolumn{3}{|c|}{20} & \multicolumn{3}{|c|}{20} \\
\hline \multirow[t]{2}{*}{ Leaflet width, mm } & $\mathrm{NC}$ & $\mathrm{RC}$ & $\mathrm{LC}$ & $\mathrm{NC}$ & $\mathrm{RC}$ & LC & $\mathrm{NC}$ & $\mathrm{RC}$ & LC \\
\hline & 29 & 29 & 29 & 27 & 29 & 29 & 27 & 29 & 29 \\
\hline \multirow[t]{2}{*}{ Leaflet length, mm } & $\mathrm{NC}$ & $\mathrm{RC}$ & $\mathrm{LC}$ & $\mathrm{NC}$ & $\mathrm{RC}$ & LC & $\mathrm{NC}$ & $\mathrm{RC}$ & LC \\
\hline & 29 & 29 & 29 & 27 & 29 & 29 & 27 & 29 & 29 \\
\hline
\end{tabular}

$N C$, Noncoronary; $R C$, right coronary; $L C$, left coronary.

tested our device explicitly in a porcine model of this procedure, we have used the device in Dacron tube grafts anastomosed just distal to the STJ and produced clear images of the loaded valve. We expect the device would be quite helpful in establishing, before coming off bypass, that the root is sized appropriately for the native valve.

A third important feature of the repaired valve is symmetry, and published studies associate coaptation asymmetry with early reoperation for AR in aortic valve repair. ${ }^{10}$ In study 2 , the gross asymmetry of our porcine model of AR was readily observable as was the restoration of symmetry after repair. In fact, our device even enabled us to discern the subtle asymmetry of the normal pig aortic valve, including the skew of the central point of coaptation toward the noncoronary sinus and the smaller relative size of the noncoronary leaflet. This observation is in agreement with published work that compared normal porcine and human aortic valves and showed that the human valve is also slightly asymmetrical but skewed toward the left coronary sinus. ${ }^{11}$

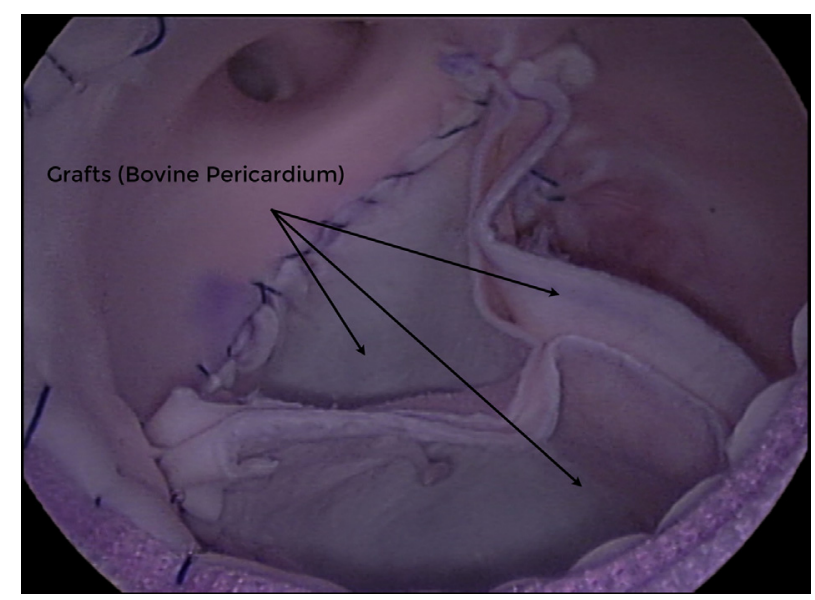

FIGURE 5. Endoscopic view showing porcine aortic valve that has been reconstructed by replacing all 3 leaflets with grafts cut from bovine pericardium.
Although the device and procedure were developed to be effective and safe in a clinical setting, there are implementation details that require further development. For example, surgeons typically transect the aorta quite close to the STJ, however if the device is secured too inferiorly in the aorta, it will not allow the STJ to dilate to its true diastolic dimension under pressure. This can distort the closed valve configuration and mislead the surgeon. Preliminary experiments have shown that the aorta can be transected within $1 \mathrm{~cm}$ of the STJ when the device diameter is well matched to the STJ diameter, however, further study is needed to ascertain how close to the STJ the device can be secured without inhibiting proper dilation of the valve root. A related issue is how to determine the optimal outside diameter of the device for a given diameter aorta. The degree to which the securing of the device in the aorta distorts the closed valve depends on the size of the device and how close to the root it is positioned, and this relationship needs to be more fully understood and described. Another issue to address is the method of securing the device in the transected aorta. We achieved good results using plastic cable ties and large silk sutures, but we need to ensure that these methods to not damage the endothelium of the aorta. A final limitation of our study concerns the snaring of the coronary arteries to allow the aortic root to maintain constant pressure during visualization. In many patients, including reoperations, dissection and snaring of the coronaries would be time-consuming and risky. We have explored alternative methods for temporarily occluding coronary blood flow including the use of plugs that are tethered for easy retrieval as well as the use of temporary occlusion clips, but these require further development before clinical deployment of our device is feasible. Another alternative is to leave the coronaries open and quickly inspect the closed valve during transient infusion of cardioplegia, although this approach would not allow the more time-consuming procedures of coaptation measurement to be performed. 


\section{CONCLUSIONS}

Our experimental prototype and pilot animal studies demonstrate the promise of a device and method for pressurizing and visualizing the aortic valve intraoperatively during surgical repair. A more refined, clinically suitable version of the device has potential to lower the barrier to better aortic valve repair outcomes and more widespread adoption of repair over replacement.

\section{Webcast}

You can watch a Webcast of this AATS meeting presentation by going to: https://aats.blob.core.windows.net/media/ 18AO/27-GramEast-0610-berra-q\%26a-v2.mp4.

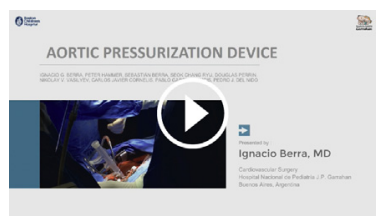

\section{Conflict of Interest Statement}

Authors have nothing to disclose with regard to commercial support.

\section{References}

1. Baird CW, del Nido PJ. Complex aortic valve disease in children. Oper Tech Thorac Cardiovasc Surg. 2009;14:253-63.

2. Jonas RA. Aortic valve repair for congenital and balloon-induced aortic regurgitation. Pediatr Card Surg Annu. 2010;13:60-5.

3. Carpentier A, Adams DH, Filsoufi F. Carpentier's Reconstructive Valve Surgery: From Valve Analysis to Valve Reconstruction. Maryland Heights, Mo: Saunders/ Elsevier; 2010.

4. Sperling JS. Apparatus and method for simulation of diastole and visualizing the diastolic state of an aortic valve and root during cardiac surgery. US Patent Application Publication, US 2011/0071351 A1, March 24, 2011.

5. Augoustides JG, Szeto WY, Bavaria JE. Advances in aortic valve repair. J Cardiothorac Vasc Anesth. 2010;24:1016-20.

6. le Polain de Waroux JB, Pouleur AC, Robert A, Pasquet A, Gerber BL, Noirhomme $\mathrm{P}$, et al. Mechanisms of recurrent aortic regurgitation after aortic valve repair: predictive value of intraoperative transesophageal echocardiography. JACC Cardiovasc Imaging. 2009;2:931-9.

7. Ozaki S, Kawase I, Yamashita H, Uchida S, Nozawa Y, Matsuyama T, et al. Aortic valve reconstruction using self-developed aortic valve plasty system in aortic valve disease. Interact Cardiovasc Thorac Surg. 2011;12:550-3.

8. Hammer PE, Berra I, del Nido PJ. Surgical repair of congenital aortic regurgitation by aortic root reduction: a finite element study. J Biomech. 2014;48:3883-9.

9. Ozaki S, Kawase I, Yamashita H, Uchida S, Nozawa Y, Takatoh M, et al. A total of 404 cases of aortic valve reconstruction with glutaraldehyde-treated autologous pericardium. J Thorac Cardiovasc Surg. 2014;147:301-6.

10. Stern KW, White MT, Verghese GR, del Nido PJ, Geva T. Intraoperative echocardiography for congenital aortic valve repair: predictors of early reoperation. Ann Thorac Surg. 2015;100:678-85.

11. Sim EK, Muskawad S, Lim CS, Yeo JH, Lim KH, Grignani RT, et al. Comparison of human and porcine aortic valves. Clin Anat. 2003;16:193-6.

Key Words: aortic valve repair, device, pressurize, viewer 